\title{
Hipotermia selectiva cefálica de baja gama en encefalopatía hipóxico-isquémica severa en un recién nacido
}

\author{
Ricardo Ávila-Reyes, Mariana Herrera-Pen, Carlos Iván Salazar-Cerda \\ y NORA InÉS VeLÁZQUEZ-QUINTANA \\ Unidad de Cuidados Intensivos Neonatales, Hospital Civil Dr. José Macías Hernández, Ciudad Victoria, Tamps., México
}

\begin{abstract}
RESUMEN
Introducción: La hipotermia selectiva cefálica se emplea para el recién nacido con encefalopatía hipóxico-isquémica de moderada a severa y demuestra que la conjugación discapacidad/muerte disminuye notablemente. Caso clínico: Recién nacido con asfixia severa al nacer, con Apgar de 1-4 y que ameritó maniobras de resucitación neonatal. Gasometría con $\mathrm{pH}$, bicarbonato $\left(\mathrm{HCO}_{3}\right)$ de 11.9, exceso de base (EB-27) y convulsiona a los pocos minutos de nacer. Se decide realizar hipotermia selectiva cefálica mediante dispositivos (gorros) con gel congelado (ice Fast ${ }^{\circledR}$ ) y se llevó a hipotermia de $34-35^{\circ} \mathrm{C}$ por $72 \mathrm{~h}$. Presentó hiponatremia, hipocalcemia e hipercalemia durante la hipotermia. Sale de la hipotermia y a los 11 días se extuba y se alimenta por sonda, y posteriormente por succión. Egresa al mes de edad y se le diagnostica parálisis cerebral motora a los 8 meses. Discusión: Se efectuó hipotermia selectiva cefálica de «baja gama» por no contar con el equipo específico para el enfriamiento cefálico. La discapacidad motora fue la única secuela determinada a los 8 meses de edad. Es posible realizar hipotermia selectiva cefálica en centros hospitalarios de segundo nivel.
\end{abstract}

Palabras clave: Hipotermia cefálica. Encefalopatía hipóxico-isquémica. Recién nacido.

\section{ABSTRACT}

Introduction: Selective cephalic hypothermia is used in newborns with moderate to severe hypoxic-ischemic encephalopathy, demonstrating a marked decrease in disability / death. Clinical case: Female newborn with severe asphyxia at birth, Apgar of 1-4, needed neonatal resuscitation, arterial blood gas $\mathrm{pH} 6.3, \mathrm{HCO}_{3}$ 11.9, EB-27, convulsed within a few minutes of birth. Selective cephalic hypothermia was performed by means of devices (caps) with frozen gel (ice Fast ${ }^{\circledR}$ ); the hypothermia was of $34-35{ }^{\circ} \mathrm{C}$ for 72 hours. She presented hyponatremia, hypocalcemia and

\section{Correspondencia:}

*Ricardo Ávila-Reyes

Hospital Civil Dr. José Macías Hernández

21 y 22 Méndez, 502

Zona Centro

Date of reception: $15-05-2018$

C.P. 87000 Ciudad Victoria, Tamps., México

Date of acceptance: $26-11-2018$

E-mail: avilareyes@ @otmail.com

DOI: 10.24875/HMCM.18000128 
hyperkalemia during hypothermia. Hypothermia was removed and after 11 days the baby was extubated. She was fed by orogastric tube and later by suction, removing the tube at one month of age. The baby was diagnosed with motor cerebral palsy at 8 months of age. Discussion: Selective "low range" cephalic hypothermia was performed because there was no specific equipment for cephalic cooling. Motor disability was the only sequel identified at 8 months of age. It is possible to perform selective cephalic hypothermia in second-level hospitals. (Hosp Med Clin Manag. 2018;11:204-10)

Corresponding author: Ricardo Ávila-Reyes, avilareyes@ hotmail.com

Key words: Cephalic hypothermia. Hypoxic-ischemic encephalopathy. Newborn.

\section{INTRODUCCIÓN}

«La encefalopatía hipóxico-isquémica es una de las causas más frecuentes de mortalidad o discapacidad neurológica en recién nacidos de término o cercanos al mismo. El tratamiento hasta la actualidad fue sólo de soporte, sintomático, con medidas generales para mantener la homeostasis, no existiendo ningún tratamiento específico. Simplemente tratar de evitar que el paciente fallezca y esperar en los supervivientes las secuelas que la injuria del sistema nervioso central (SNC) determinara. En los últimos años, felizmente comenzamos a ver una luz en la penumbra. La hipotermia corporal o encefálica selectiva está demostrando, tanto en los ensayos clínicos como en el aquí presentado, que este tratamiento reduce el daño neurológico a mediano plazo e incluso aumenta las posibilidades de vida sin secuelas en pacientes que sufrieron encefalopatía asfíctica perinatal moderada y severa, siendo más evidente en el primer caso. Aunque sigue siendo un tratamiento experimental, muchas unidades de alta complejidad lo han adoptado como tratamiento de rutina y esto se está diseminando con rapidez en el mundo, a pesar de que existen aún interrogantes por resolver. La realidad es que los resultados son promisorios y los riesgos aparentemente bajos. Junto a este noble avance, los expertos coinciden en que deben explorarse otras herramientas con las que contar, ya que aun con hipotermia, alrededor de un $40 \%$ de los pacientes con encefalopatía de moderada a severa igualmente fallecerán o tendrán discapacidad neurológica. Siendo el daño neurológico un proceso complejo que tiene varios caminos que conducen al mismo, el uso de terapias combinadas de neuroprotección en estudio, tanto farmacológicas como de neurorregeneración, probablemente mejorarán los resultados en los próximos años. Por lo menos ya no somos meros espectadores de una tragedia inevitable» $(\mathrm{sic})^{1}$. Esto concluye el autor y concreta o resume lo que en la gran mayoría de los metaanálisis y/o ensayos controlados en pacientes con encefalopatía hipóxico-isquémica sometidos a hipotermia dan como resultados alentadores. El presente caso versa de un recién nacido con encefalopatía hipóxico-isquémica severa, en el que se inició hipotermia selectica cefálica bajo los criterios del Cool Cap TriaR, y que sobrevivió con secuela neurológica de tipo parálisis cerebral motora.

\section{CASO CLÍNICO}

Recién nacido, de sexo femenino, hija de madre de 16 años, con una gesta, sin enfermedades cronicodegenerativas ni toxicomanías y fecha de última menstruación (FUM) el 28/7/11. La madre asistió a ocho consultas prenatales y desarrolló infección de las vías urinarias en el último trimestre, la cual fue controlada satisfactoriamente. Ingresó con trabajo de parto activo y se pasó a expulsión, en la que la bebé tardó más de 50 min en nacer. Nació sin esfuerzo respiratorio, sin Ilanto, con flacidez total, sin tono muscular, cianótica y con frecuencia cardíaca (FC) de $60 \mathrm{lpm}$. Fue colocada en cuna térmica. Se realizó reanimación cardiopulmonar, ventilando con bolsa y máscara con oxígeno al 100\%. Posteriormente fue intubada. No se cuenta con pulsioxímetro. A los $30 \mathrm{~s}$ es revisada y mantiene la $\mathrm{FC}$ en $70 \mathrm{lpm}$. Se sigue con las maniobras de reanimación y $30 \mathrm{~s}$ después aumenta la FC a 90 lpm. La bebé continúa sin esfuerzo respiratorio, cianosis central, pupilas mióticas y flacidez muscular. Se continúa la ventilación con presión positiva por más de $10 \mathrm{~min}$, subiendo a 140 lpm. Aparece acrocianosis, persiste sin tono muscular y es transferida a la Unidad de Cuidados Intensivos Neonatal. Apgar de 1-4-7. En los primeros 5 min de vida se tomó gasometría arterial, $\mathrm{pH}$ de 6.30, $\mathrm{pCO}_{2}$ de 106, $\mathrm{pO}_{2}$ de 10.7, $\mathrm{HCO}_{3}$ de 11.9, EB27 y Sat $\mathrm{O}_{2}$ del $38.8 \%$. A los pocos minutos de estar en la UCIN desarrolló convulsiones tónico-clónicas en cara y extremidad superior derecha. Se inició manejo 


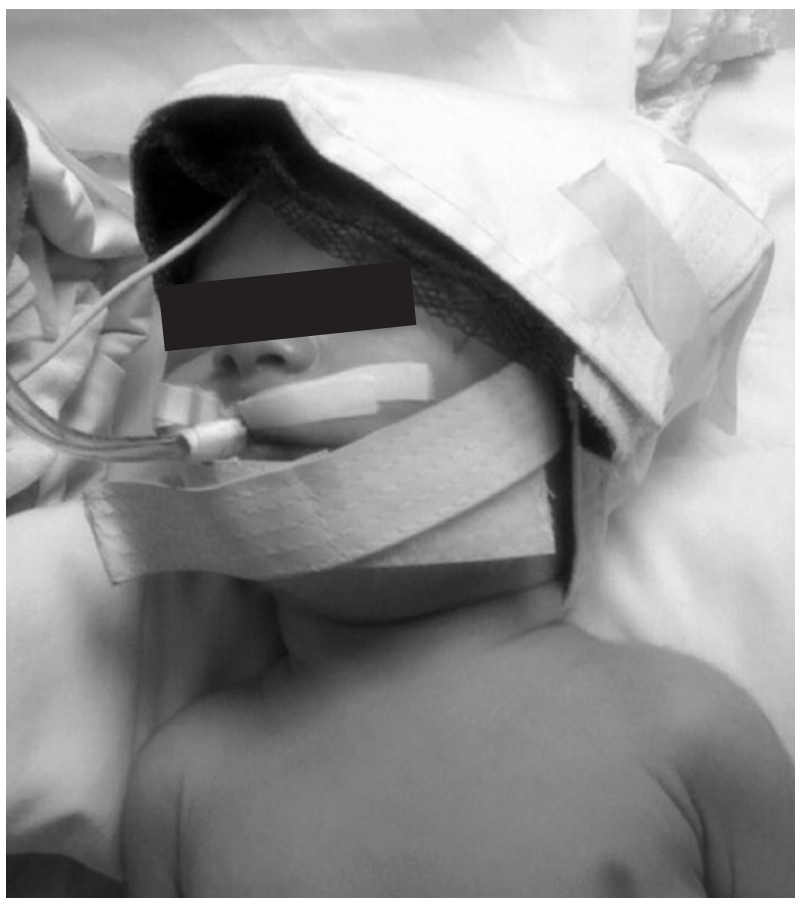

Figura 1. Dispositivo cefálico (gorro) en vinyl para la hipotermia cefálica.

farmacológico con midazolam y fenitoína, y se ajustaron los parámetros al ventilador: presión inspiratoria de 20 $\mathrm{cmH}_{2} \mathrm{O}$, PPFE de $4 \mathrm{cmH}_{2} \mathrm{O}, 35$ de frecuencia respiratoria y $\mathrm{FiO}_{2}$ del $40 \%$. A las $4 \mathrm{~h}$ de vida se obtiene el consentimiento familiar y es ingresada en el protocolo de hipotermia cefálica selectiva mediante unos dispositivos manufacturados en tela de plástico con malla en su interior (Fig. 1), para contener bolsas de gel refrigerante (ice Fast $^{\circledR}$ ) no congeladas, solamente frías, a fin de mantener la temperatura de $34-35^{\circ} \mathrm{C}$ rectal por $72 \mathrm{~h}$. Se mantuvo bajo incubadora de calor radiante mediante servocontrol abdominal, ajustándose cuanto fue necesario para mantener la temperatura rectal a $34^{\circ} \mathrm{C}$. El peso al nacer fue de $3,400 \mathrm{~g}$, la estatura de $50 \mathrm{~cm}$ y el perímetro cefálico de $36 \mathrm{~cm}$. Nació a las 41 semanas de gestación, según la valoración de Capurro. Se clasificó en estadio III de la escala de Sarnat. El promedio de la temperatura durante el primer día fue de $34.5^{\circ} \mathrm{C}$ (rango: $34-35.1^{\circ} \mathrm{C}$ ) y la FC en promedio fue de 106 lpm (rango: 104-136 lpm). Presentó temblores en las extremidades y pupilas con tendencia a la miosis. Se decidió inducir con tiopental a razón de $1 \mathrm{mg} / \mathrm{kg} / \mathrm{h}$. La biometría hemática mostró: leucocitosis de 23,000, un 53\% de granulocitos, 218,000 plaquetas, $\mathrm{Hb}$ de 17, Hto de 49.1, Na sérico de 130 $\mathrm{mmol} / \mathrm{l}$ y potasio de $4 \mathrm{mmol} / \mathrm{l}$. El manejo ventilatorio permaneció sin cambios, llevando a valores gasométricos normales y saturación de oxígeno por pulsioximetría del
88-93\%. Elevación de transaminasas atribuida a hipoxia. Pruebas de función renal y hepática normales. Se inició nutrición parenteral. El segundo día de hipotermia el promedio de FC fue de 122 Ipm (rango: 112-136 lpm) y la temperatura de $34.6{ }^{\circ} \mathrm{C}$. Desarrolló hiponatremia de $121 \mathrm{mmol} / \mathrm{l}$, hipocalcemia de $6 \mathrm{mg} / \mathrm{dl}$, por lo que recibió calcio desde el primer día a $100 \mathrm{mg} / \mathrm{kg} / \mathrm{día}$, que se incrementó a $200 \mathrm{mg} / \mathrm{kg} / \mathrm{día}$. Se calculó el sodio para llevarlo a $\mathrm{Na}$ ideal de $125 \mathrm{mmol} / \mathrm{l}$. El potasio resultó en $4 \mathrm{mmol} / \mathrm{l}$. Los leucocitos descendieron a 12,000, con predominio del $67 \%$ de granulocitos y plaquetas de 128,000; Hb de 15.7 y Hto de 45.2. El ultrasonido transfontanelar reportó parénquima cerebral con ligero aumento de la ecogenicidad de manera difusa, sin evidencia de masas, edema ni hemorragias, la línea media sin desplazamiento y los ventrículos laterales parcialmente colapsados, con un diámetro de $5 \mathrm{~mm}$. La función renal era normal. Neurológicamente hipotónica e hiporreactiva, con las pupilas midriáticas con poca respuesta a la luz.

El tercer día persistía con hiponatremia de $120 \mathrm{mmol} / \mathrm{l}$ y potasio de $7.1 \mathrm{mmol} / \mathrm{l}$. Se retiró el potasio de las soluciones y se inició manejo con furosemida para la hipercalemia. Calcio sérico de $7.9 \mathrm{mg} / \mathrm{dl}$. Al llegar a las $70 \mathrm{~h}$ de vida tenía una temperatura promedio de $34.1^{\circ} \mathrm{C}$ y una FC promedio de $118 \mathrm{lpm}$. Finalizó el protocolo de hipotermia a las $72 \mathrm{~h}$, se retiró el dispositivo cefálico y se incrementó gradualmente la temperatura $0.5^{\circ} \mathrm{C}$ por hora. La biometría hemática incrementó a 27,000 los leucocitos, con 178,000 plaquetas, $\mathrm{Hb}$ de 16.5 , Hto de 48.4 y $\mathrm{Na}$ sérico de $116 \mathrm{mmol} / \mathrm{l}$. Pese a la corrección, el potasio era de $6.6 \mathrm{mmol} / \mathrm{l}$ y el calcio de $7.8 \mathrm{mg} / \mathrm{dl}$. Procalcitonina de $15.93 \mathrm{ng} / \mathrm{ml}$. Se indicó cefotaxima. El manejo del ventilador continuó, ajustándose a los resultados gasométricos, teniendo a estas alturas $18 \mathrm{cmH}_{2} \mathrm{O}$ de presión pico, 4 de PPFE, 45 respiraciones por minuto y $\mathrm{FiO}_{2}$ al $60 \%$. Del cuarto al séptimo día desarrolló coagulopatía, al tener TP de 132", TPT de 83" y plaquetas de 69,000. No fue posible realizar prueba de fibrinógeno. Leucocitos en 7,000, 61 granulocitos, $\mathrm{Hb}$ de 10.7 y hematocrito (HTO) de 31. Se transfunden concentrado plaquetario, globulares y plasma fresco, además de vitamina K. El sodio sérico se corrige a $119 \mathrm{mmol} / \mathrm{l}$. La bebé continúa sin respuesta a los estímulos, con hipotonía generalizada e hiporreflexia. Termina con Na sérico de $132 \mathrm{mmol} / \mathrm{l}$. Se suspenden el tiopental y el midazolam. Continúa con asistencia mecánica a la ventilación y sin automatismo respiratorio. El ultrasonido cerebral reporta rastreo axial, sagital y coronal de la fontanela anterior y lateral, con los 


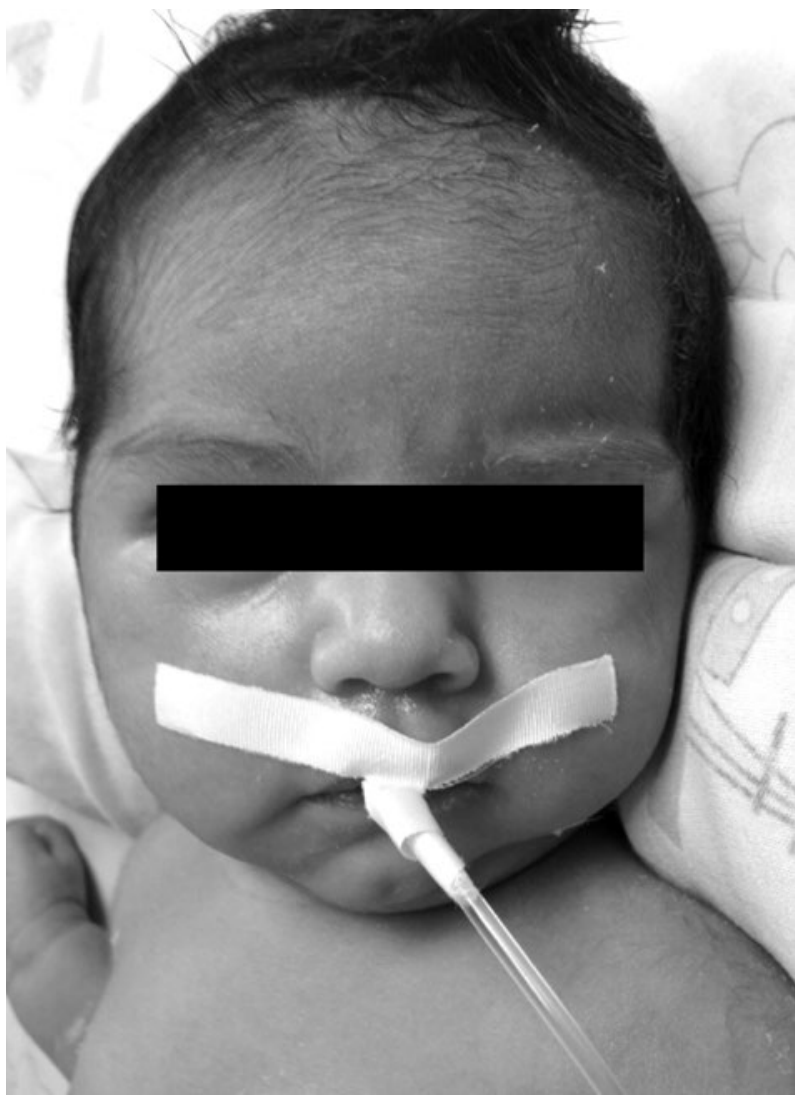

Figura 2. Aspecto clínico de la paciente a los 25 días de vida.

siguientes hallazgos: parénquima cerebral con un ligero aumento de la ecogenicidad de manera difusa, sin evidencia de masas, edema ni hemorragias; línea media sin desplazamiento; ventrículos laterales parcialmente colapsados, con un diámetro de $5 \mathrm{~mm}$, y cambios por hipoxia cerebral. Al octavo día, con las pupilas discretamente refléxicas a la luz, inicia respiraciones irregulares y movimientos de las extremidades y cara tipo temblores finos a gruesos; hiperreflexia, con aumento del tono muscular de las extremidades. Los electrólitos séricos normales e hiperglicemia en dos ocasiones, por lo que se corrige el ajuste del aporte de glucosa. Se solicita intervención para estimulación temprana. Durante el noveno y décimo día mejora las respiraciones, por lo que se deja de asistir su ventilación de modo controlado y pasa a ventilación mandatoria intermitente sincronizada, y posteriormente a ventilación de soporte con presión. Disminuyen los movimientos de las extremidades y los temblores de los miembros y la cara, las pupilas son isocóricas y responden a la luz, apertura ocular espontánea, reflejos con respuesta débil, no succión y no reflejo de Moro. Hay mayor movimiento de sus extremidades y empieza a «luchar» con el tubo endotraqueal. Se inicia alimentación

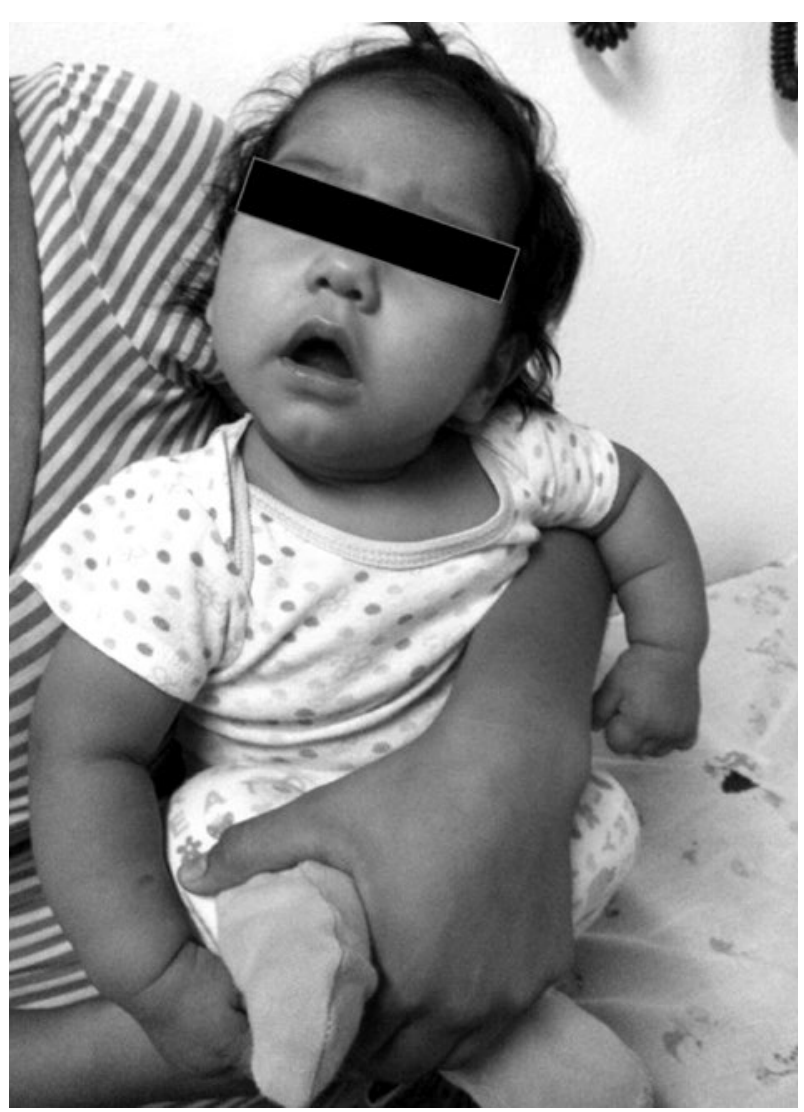

Figura 3. Aspecto clínico de la paciente a los 8 meses de edad.

enteral mínima por sonda orogástrica con fórmula elemental. En los días 11 y 12 se extuba y pasa a casco cefálico por $2 \mathrm{~h}$, que posteriormente se retira. Mantiene buen automatismo respiratorio y mirada conjugada, facies normal, y no hay movimientos anormales de la cara ni de las extremidades; el tono muscular es normal-discretamente débil en las extremidades superiores e inferiores, tono débil del cuello y continúa sin succión. Se aumenta la cantidad de alimentación por sonda y se empieza a disminuir la nutrición parenteral total. Succión no nutritiva. Permaneció en vigilancia porque no tenía succión efectiva y fue alimentada con sonda orogástrica, sin datos de infección (Fig. 2). Se le realizaron ejercicios de estimulación temprana. Estuvo hospitalizada 31 días, y cuando egresa pesa 3,940 g y mide $54 \mathrm{~cm}$, tiene buena succión y deglución, buen tono de cuello y tronco, y reflejos normales. Fue enviada a tercer nivel para seguimiento neurológico, electroencefalograma (EEG) y estimulación temprana. Regresó a consulta al hospital hasta los 8 meses de edad, debido a que recibió tratamiento en el hospital de referencia. En la exploración física, sostiene la cabeza y el tronco, las extremidades son flexibles, con fuerza normal y tono discretamente aumentado, 
se sienta con apoyo, deglute papillas y tiene succión normal (Fig. 3). Se solicitó la revisión por un neurólogo externo (pues el hospital no cuenta con uno propio), quien clínicamente consideró que la paciente presentaba parálisis cerebral motora y que debía continuar en el programa de estimulación temprana. Continúa siendo visitada en el Servicio de Neurología del hospital de referencia.

\section{DISCUSIÓN}

La EHI sigue siendo en la actualidad un problema para la sociedad en todo el mundo, tanto por su frecuencia, del 3.5\% (3 de cada 1,000 NV en EE.UU.) en RN a término, como por la mortalidad, del $10-60 \%$ alrededor del mundo, y el $25 \%$ de secuelas neurológicas que deja en los sobrevivientes. La parálisis cerebral con causa primaria en EHI va del 15 al 18\%, con profundas consecuencias psicosociales y económicas en la familia y la sociedad ${ }^{1-3}$. La hipotermia leve para los bebés asfixiados surge a finales de 1990 como una posible intervención para la lesión cerebral que ocurre entre la fase inicial y la segunda fase de daño neuronal asociado con $\mathrm{EHI}$ (antes de las $6 \mathrm{~h}$ postasfixia). Este primer ensayo controlado aleatorio, y piedra angular de la intervención de hipotermia en neonatos, fue el Cool-Cap Trial. El ensayo reclutó a 234 lactantes con evidencia de asfixia y la consiguiente encefalopatía entre julio de 1999 y enero de 2002. Los criterios de inclusión del estudio Cool-Cap son los que se emplearon para el protocolo de hipotérmica cefálica selectiva en nuestro hospital ${ }^{2}$. Se inició dentro de las primeras $6 \mathrm{~h}$ el enfriamiento cefálico debido a la estabilización del paciente y la explicación detallada del protocolo a los familiares, que dieron el consentimiento para someter a la niña a hipotermia. En la localidad no había ningún centro de referencia para la hipotermia cefálica, por lo cual se diseñaron los gorros y el protocolo apegado al ensayo Cool-Cap Trial. Desde la primera serie de casos de asfixia perinatal tratada con hipotermia, la gran mayoría de los estudios demuestran que la ventana terapéutica para reducir e inhibir el daño hipóxico-isquémico es dentro de las primeras $6 \mathrm{~h}$ posteriores al evento de asfixia, y se considera que la duración de la fase latente es inversamente proporcional a la severidad del daño ${ }^{1-4}$. Por ello, se exhorta a que en casos de asfixia moderada el retraso del enfriamiento pueda ser de 0.5 a $3 \mathrm{~h}$, pero en casos graves no debe retardarse más allá de 15-45 min después del daño. Según la experiencia de varios modelos de hipotermia en adultos, se concluye que la duración requerida del enfriamiento va en relación con el tipo y la gravedad de la agresión primaria, lo que sugiere que dicho enfriamiento actúa a través de la supresión sostenida de los eventos citotóxicos en la fase secundaria de la falla energética. En segundo lugar, la ventana de oportunidad de enfriamiento después de la asfixia (o daño focal reversible) puede ser de hasta $6 \mathrm{~h}$ o incluso más en algunas circunstancias; el grado de neuroprotección disminuye progresivamente si el enfriamiento se inicia más de unas pocas horas después del daño $0^{5-7}$. Las evidencias que sustentan la neuroprotección hipotérmica incluyen una disminución en la tasa metabólica con disminuciones paralelas en el consumo de $\mathrm{O}_{2}$ y producción de $\mathrm{CO}_{2}$, pérdida reducida de fosfatos de alta energía durante hipoxia-isquemia y durante la falla secundaria de energía cerebral actúa en la excitotoxicidad reducida, reducción de la producción de especies de oxígeno reactivo, proteína de preservación de la síntesis, disminución del edema, activación microglial, modulación de la cascada inflamatoria y un cambio en la señalización proapoptótica y antiapoptótica ${ }^{1-6}$. El grupo colaborativo Cochrane revisó un total de 11 estudios con 1,505 neonatos con asfixia moderada-severa, y el manejo con hipotermia resultó con significancia estadística al reducir de manera importante la mortalidad y el retraso del neurodesarrollo a los 18 meses de vida. En nuestro caso, por el pH de la gasometría, el test de Apgar y las convulsiones tempranas, consideramos que desarrolló EHI severa y sobrevivió. Resulta incierto decir si podría haber sobrevivido sin la hipotermia; sin embargo, el daño neurológico se minimizó, ya que pudo haber sido más catastrófico. Se considera que la acción neuroprotectora de la hipotermia es menos efectiva en la EHI severa debido al periodo de latencia más corto, estando acelerada la necrosis neuronal de la materia gris, que se asocia a daño cerebral a distintos grados. En nuestro caso, consideramos que la acción protectora de la hipotermia fue benéfica, ya que las secuelas neurológicas pudieron haber sido mayores. Se recomienda que a los pacientes sometidos a hipotermia se les continúe visitando por largo tiempo para evaluar su evolución, y que en la hipotermia, para ser efectiva, debe otorgarse un nivel alto de soporte de cuidados intensivos neonatales, dado que no todos los centros son capaces de realizar terapia de hipotermia ${ }^{4}$. El caso que nos compete se atendió en un hospital civil de segundo nivel, pero, aunque cuenta con Unidad de Cuidados Intensivos Neonatal con neonatólogos en todos los turnos, se procedió y revisó conjuntamente el 
protocolo y las adecuaciones respecto al diseño de los dispositivos cefálicos. Se emplearon bolsas de gel frío en el interior del gorro en un aditamento especial que no hiciera contacto con el epicráneo, pero que sí fuera capaz de enfriar, monitorizando el interior del gorro con termómetro digital para el cambio del gel cuando la temperatura se elevara y así garantizar la hipotermia rectal entre 34 y $35{ }^{\circ} \mathrm{C}$, en tanto que se ajustaba el servocontrol abdominal para mantenerlo en esta temperatura.

Se reporta que posibles efectos adversos de la hipotermia son hipotensión, QT prolongado, trombocitopenia, prolongación en tiempos de coagulación, lesiones del cuero cabelludo, escleroderma, hipoglicemia, bradicardia y alteraciones electrolíticas como hipercalemia ${ }^{1-5}$. En nuestro caso se presentó FC baja, hiponatremia e hipercalemia, que se manejaron oportunamente en cada evento adverso atribuido al enfriamiento, logrando completar las $72 \mathrm{~h}$ de enfriamiento, con un promedio de $34.6^{\circ} \mathrm{C}$. Los tiempos de coagulación prolongados y la plaquetopenia se presentaron después de la hipotermia, y no durante ésta. Consideramos que, al no contar con un dispositivo como el Olimpic Cool Cap ${ }^{\mathrm{TM}}$, que mantiene de forma permanente el enfriamiento, con los gorros utilizados tuvimos rangos de $34-35.1^{\circ} \mathrm{C}$; sin embargo, sí lo llevamos a enfriamiento selectivo moderado la mayor parte de las $72 \mathrm{~h}$. Las complicaciones que desarrolló la paciente, por tanto, son mayormente atribuibles al enfriamiento, e hizo considerar estar en zona de seguridad de enfriamiento selectivo cefálico moderado. Hace dos décadas, en los albores de la hipotermia selectiva cefálica, la temperatura rectal se fijaba en $\geq 35^{\circ}$ y las complicaciones de tal enfriamiento no se desarrollaban significativamente en cuanto a bradicardia, hipoglicemia y trastornos electrolíticos. Pese a no llegar a los $34^{\circ} \mathrm{C}$, como se establece actualmente en la hipotermia moderada (32-34 ${ }^{\circ}$ ), la evolución neurológica fue normal en el grupo de mayor enfriamiento. Caso contrario son los de hipotermia mínima y los que no se sometieron a hipotermia, que presentaron mayor secuela neurológica a los 6-12 meses $^{7}$. En otros estudios controlados de enfriamiento selectivo cefálico realizados en China, llevados a hipotermia a $34^{\circ} \mathrm{C}$, con lectura de temperatura nasofaríngea e hipotermia corporal moderada de $34.5-35^{\circ} \mathrm{C}$, hubo diferencias en la incidencia combinada de muerte y discapacidad mayor, siendo más bajo en el grupo de enfriamiento selectivo cefálico que en el grupo de control, que mantuvo temperatura rectal o nasofaríngea de $36-36.5^{\circ} \mathrm{C}^{8}$. Las complicaciones como arritmia, hemorragia, alteraciones electrolíticas, hipoglicemia y plaquetopenia sí ocurrieron, pero no tuvieron diferencias estadísticamente significativas entre ambos grupos. Por tanto, es relevante conocer los componentes de los tratamientos de hipotermia: tiempo de iniciación, duración y profundidad de enfriamiento, ritmo de recalentamiento y modo de enfriamiento (que puede ser corporal, selectivo cefálico, cefálico y corporal) ${ }^{9}$, en donde se considera aún la ventana terapéutica dentro de las primeras $6 \mathrm{~h}$ de vida, y prolongación hasta $72 \mathrm{~h}$ de enfriamiento, $32-34{ }^{\circ} \mathrm{C}$ de temperatura, con ritmo de recalentamiento de 0.2 a $0.5^{\circ} \mathrm{C}$ por hora y mayormente enfriamiento cefálico, que es lo que la mayoría de estudios describen en sus estudios ${ }^{1-11}$.

Respecto al recalentamiento corporal, algunos estudios comentan recalentar de manera espontánea ${ }^{8}$, pero otros describen que se debe incrementar $0.5{ }^{\circ} \mathrm{C}$ por hora, ya que la fase de recalentamiento no debe ser rápida por el riesgo de hemorragia-1-6,9,10. En nuestro caso optamos por ir cambiando los paquetes de gel cada vez menos congelados hasta estar frescos y modificando el servo de la incubadora para dar una elevación aproximada de $0.2-0.5{ }^{\circ} \mathrm{C}$ por hora. El estudio TOBY, con enfriamiento total corporal, no mostró reducción significativa en la combinación muerte/discapacidad entre los pacientes que se enfriaron versus los que no se sometieron a hipotermia, pero sí mostró mejoría significativa en varios resultados neurológicos entre los sobrevivientes ${ }^{10}$. Es importante remarcar que los centros terciaros son lo que habitualmente cuentan con equipamiento para un enfriamiento servo controlado, ya que son costosos pero accesibles a este tipo de instituciones de salud. En nuestro caso, un hospital civil de segundo nivel de atención, a pesar de existir un hospital de tercer nivel, éste no contaba con un equipo de enfriamiento diseñado exclusivamente para enfriamiento de recién nacidos. No contamos con EEG ni neurólogo, a pesar de que en algunos protocolos se solicita EEG como criterio para someter a hipotermia al neonato, por lo que el EEG no debe ser un condicionante absoluto $^{11}$. Se considera que en los centros que no cuenten con equipo especializado para la hipotermia del recién nacido, éste se deje en hipotermia pasiva, es decir, no aplicar medidas de calentamiento activo al neonato con asfixia. En un neonato sano con hipotermia pasiva, la temperatura puede llegar a descender $1.5^{\circ} \mathrm{C}$ en media hora, y en un recién nacido con asfixia se requiere reducir hasta $3{ }^{\circ} \mathrm{C}$ con asfixia moderada y ventilación espontánea, evitando el sobreenfriamiento 
$\left(<32{ }^{\circ} \mathrm{C}\right)$, para lo cual se conjuga con encendido de la incubadora a mínima intensidad. La hipotermia pasiva de fácil acceso para hospitales emisores puede dar lugar a latencia hasta $15 \mathrm{~h}$ para su envío a un hospital que cuente con equipamiento para hipotermia, enviado siempre en incubadora de transporte apagado. Sólo en casos especiales se pueden emplear gel frío/hielo en el perímetro del cuerpo del neonato a unos $10 \mathrm{~cm}$, para evitar quemaduras por frío, y retirar cuando la temperatura sea inferior a $35{ }^{\circ} \mathrm{C},\left(1{ }^{\circ} \mathrm{C}\right.$ por encima de la temperatura blanco). En nuestro caso, se decidió optar por el enfriamiento de «baja gama», que se describe como el empleo de botellas de agua, packs de gel frío, gorros de hielo y ventiladores, etc. ${ }^{11}$. En este tipo de modalidad se refiere tener un control difícil de la hipotermia y es un arduo trabajo de enfermería, y, efectivamente, se tuvo mucha carga asistencial del equipo de enfermería.

Para el caso que nos compete se diseñaron unos dispositivos (gorros) con protección de malla para evitar el contacto directo de la bolsa de gel con el cuero cabelludo y así no ocasionar lesiones. Aunque se considera como opción el enfriamiento de «baja gama» para países de escasos recursos, precisa de mucho equipo técnico, por lo que se insta a tener centros de referencia que brinden la terapia de hipotermia protocolizada y revisada por pares, y después capacitarse por el proveedor del dispositivo de enfriamiento. Los pediatras en hospitales emisores juegan un papel importante en la determinación del acceso a la hipotermia terapéutica para los bebés en riesgo. Se debe reconocer oportunamente a los bebés en riesgo, estabilizarlos adecuadamente en atención al mantenimiento de la normotermia o hipotermia pasiva, pero, sobre todo, tener comunicación con los centros de referencia que cuenten con recursos para proporcionar esta terapia, sin soslayar la atención adecuada en el transporte, que contribuirá a los beneficios de esta intervención. Actualmente, en el hospital de referencia de la entidad ya se cuenta con el Olympic Medical Cool Care System ${ }^{\circledR}$, por lo que allí se envían a los neonatos que cumplen los criterios para la hipotermia cefálica selectiva. Finalmente, el caso que nos compete se logró someter a hipotermia y completar las $72 \mathrm{~h}$, pero la secuela fue la parálisis cerebral motora. En consecuencia, es posible realizar la hipotermia selectiva cefálica de baja gama en hospitales que no cuenten con dispositivos de enfriamiento cefálico o corporal para el recién nacido.

\section{BIBLIOGRAFÍA}

1. García-Alix A, González de Dios J. Hipotermia terapéutica en la encefalopatía hipóxico-isquémica del recién nacido. Evid Pediatr. 2010;6:144-6.

2. Selway LD. Hypoxic ischemic encephalopathy and hypothermic intervention for neonates. Adv Neonatal Care. 2010;10:60-6.

3. Hansell KJ, Ezzati M, Alonso-Alconada D, Hausenloy DJ, Robertson NJ. New horizons for newborn brain protection: enhancing endogenous neuroprotection. Arch Dis Child Fetal Neonatal. 2015;100:F541-52.

4. Silveira RC, Procianoy RS. Hypothermia therapy for newborn with hypoxicischemic encephalopathy. J Pediatr (Rio J). 2015;91(6 Suppl 1):S78-83.

5. Sahni R, Sanocka UM. Hypothermia for hypoxic-ischemic encephalopathy. Clin Perinatol. 2008;38:717-34.

6. Valera M, Berazategui JP, Saa G, Olmo-Herrera C, Sepúlveda T, Buraschi MF, et al. Implementation in Buenos Aires City of a program to prevent neurological damage caused by hypoxic-ischemic encephalopathy. Therapeutic hypothermia. Arch Argent Pediatr. 2015;113(5):437-42.

7. Gunn AJ, Gluckman PD, Gunn T. Selective head cooling in newborn infant after perinatal asphyxia: A safety study. Pediatrics. 1998;102:885-92.

8. Zhou WH, Cheng GQ, Shao XM, Liu XZ, Shan RB, Zhuang DY, et al. Selective head cooling with mild systemic hypothermia after neonatal hypoxicischemic encephalopathy: A multicenter randomized controlled trial in China. J Pediatr. 2010;157:367-72.

9. Laptook A. Use of therapeutic hypothermia for term infant with hypoxicischemic encephalopathy. Pediatr Clin N Am. 2009;56:601-16.

10. Azzopardi DV, Strohm B, Edwards AD, Dyet L, Halliday HL, Juszczak E, et al. Moderate hypothermia to treat perinatal asphyxia encephalopathy. N Engl J Med. 2009;351:1349-58.

11. Blanco D, García-Alix A, Valverde E, Tenorio V, Vento E, Cabañas F. Neuroprotección con hipotermia en recién nacidos con encefalopatía hipóxicoisquémica. Guía de estándares para su aplicación clínica. An Pediatr (Barc). 2011;75(5):341.e1-20 